\title{
Efficacy and Safety of Cinobufacin Combined with Chemotherapy for Advanced Breast Cancer: A Systematic Review and Meta-Analysis
}

\author{
Jing Xu, ${ }^{1,2}$ Dongyun Li $\mathbb{D}^{1},{ }^{1}$ Kexin Du, ${ }^{1,2}$ and Jing Wang $\mathbb{D}^{1}$ \\ ${ }^{1}$ Department of Hematology and Oncology, Dongzhimen Hospital Affiliated with Beijing University of Chinese Medicine, \\ Beijing 100700, China \\ ${ }^{2}$ Beijing University of Chinese Medicine, Beijing 100029, China \\ Correspondence should be addressed to Dongyun Li; lidy0039@sina.com and Jing Wang; jwang2936@126.com
}

Received 22 July 2020; Revised 24 August 2020; Accepted 2 September 2020; Published 19 September 2020

Academic Editor: Mohd Fadzelly Abu Bakar

Copyright (C) 2020 Jing Xu et al. This is an open access article distributed under the Creative Commons Attribution License, which permits unrestricted use, distribution, and reproduction in any medium, provided the original work is properly cited.

Background. Cinobufacin is a Chinese patent medicine widely used for breast cancer in China. However, no systematic review and meta-analysis have been published to validate its effects in breast cancer treatment. We, therefore, summarize the efficacy and safety of Cinobufacin combined with chemotherapy in order to provide rigid evidence for its clinical application. Methods. By searching multiple databases incepted to December 2019, the RCTs of breast cancer patients treated with Cinobufacin were screened according to the inclusion criteria, and the meta-analysis and sensitivity analysis were conducted using RevMan5.3. Results. A total of 1163 articles were retrieved, and 16 studies were included. The total sample size was 1331 cases, including 666 cases in the treatment group receiving Cinobufacin combined with chemotherapy and 665 cases in the control group receiving chemotherapy alone. Our study found that the ORR (overall response rate) (RR=1.35, 95\% CI: [1.23, 1.49], $P<0.00001$ ), CBR (clinical benefit rate) $(\mathrm{RR}=1.14,95 \% \mathrm{CI}$ : $[1.08,1.21], P<0.00001)$, KPS scores (RR=1.98, 95\% CI: $[1.45,2.68], P<0.0001)$, and pain relief rate $(\mathrm{RR}=1.34,95 \% \mathrm{CI}$ : $[1.01,1.78] P=0.04$ of the Cinobufacin combined with chemotherapy group were better than those of the chemotherapy group, and the difference was statistically significant. Our study also discovered that the tumor markers (CA125, CA153, and CEA) in the Cinobufacin combined with chemotherapy group were lower than those in the chemotherapy group, which heterogeneity was derived from the low-quality literature included in the study, but the results were robust. In addition, in terms of safety, we found that the incidences of gastrointestinal reactions $(\mathrm{RR}=0.58,95 \% \mathrm{CI}$ : [0.48, 0.70], $P<0.00001)$, liver and kidney damage $(\mathrm{RR}=0.57,95 \% \mathrm{CI}$ : [0.38, 0.84], $P=0.004)$, and hair loss ( $\mathrm{RR}=0.61,95 \% \mathrm{CI}:[0.40,0.92]$, $P=0.02)$ in the Cinobufacin combined chemotherapy group were lower than those in the chemotherapy group, and the difference was statistically significant, but the incidences of peripheral neurotoxicity $(\mathrm{RR}=0.69,95 \% \mathrm{CI}$ : $[0.26,1.85], P=0.46)$ and myelosuppression ( $R R=0.78,95 \% \mathrm{CI}:[0.46,1.34], P=0.37$ ) in the combined group were similar to those of the chemotherapy group, and the difference was not statistically significant. Conclusions. Cinobufacin combined with chemotherapy can improve the clinical efficacy of breast cancer patients, enhance the quality of life of the patients, reduce the value of tumor markers such as CA125, CA153, and CEA, and lower the occurrence of adverse reactions such as gastrointestinal reactions, liver and kidney damage, and hair loss.

\section{Introduction}

Breast cancer is the malignant tumor with the highest female morbidity and the second highest mortality after lung cancer in the world. According to global cancer data statistics, there were more than 2 million new cases of breast cancer in 2018, accounting for $11.6 \%$ of the total number of new cancers. Among them, the incidence of breast cancer in China is as high as $36.1 / 100,000$. More than 600,000 women die from breast cancer, accounting for $6.6 \%$ of total cancer-related deaths each year $[1,2]$. Chemotherapy is one of the commonly used treatment methods for advanced breast cancer, 
but its application is limited because of its severe side effects, including gastrointestinal symptoms, myelosuppression, liver and kidney damage, etc [3].

Cinobufacin is a traditional Chinese patent medicine extracted from the skin of Bufo bufo gargarizans. Its components are toadoxin, dehydroxytoluotoxin, serotonin, amino acids, reducing sugar, arginine complex, etc. It has the functions of clearing away heat and detoxification, promoting blood circulation, and removing and resolving blood stasis [4]. In recent years, many studies have shown that Cinobufacin has antitumor effects, which may be related to its inhibition of tumor cell growth, induction of tumor cell apoptosis, and enhancement of immune function in the body [5]. Multiple meta-analyses have proved that Cinobufacin combined with chemotherapy can improve the efficacy and reduce adverse reactions in gastric cancer, liver cancer, non-small-cell lung cancer, rectal cancer, and other malignant tumors [6-9]. However, there are currently no evidence-based medicine data to demonstrate the efficacy and safety of Cinobufacin for breast cancer. Therefore, we carried out a meta-analysis of Cinobufacin based on the RCT literature of breast cancer to evaluate the efficacy and safety of its treatment and to provide guidance for the clinical application of Cinobufacin.

\section{Methods}

2.1. Protocol and Registration. First, the study was according to the statements of the Preferred Reporting Items for Systematic Reviews and Meta-Analyses (PRISMA) [10]. The protocol for this review has been registered on PROSPERO, and the registration number is CRD42020154411.

2.2. Literature Search. Our research retrieved the three major English databases of PubMed, Web of Science, Cochrane Library, and the four major Chinese databases of CNKI, WanFang, VIP, and SinoMed, with "Huachansu", "cinobufotalin", "cinobufacin", "cinobufagin", "cinobufatini", "toad skin" and "breast cancer", "breast carcinoma", and "breast tumor" as main inscriptions or keywords as well as subject words or free words. For example, the PubMed retrieval strategy was as follows: (Huachansu OR cinobufotalin OR cinobufacini OR cinobufagin OR cinobufatini OR toad skin) AND (breast cancer OR breast carcinoma OR breast tumor). The retrieval deadline is until December 2019. In addition, the references that were reviewed and included in the study were searched twice.

2.3. Inclusion and Exclusion Criteria. Our inclusion criteria include, first, randomized controlled trials published in China and abroad, regardless of language; second, all patients are confirmed as advanced breast cancer by pathology and imaging examination; third, the treatment group was treated with Cinobufacin combined with conventional chemotherapy, while the control group was treated with conventional chemotherapy; and fourth, inclusion of research outcome indicators is required (contains one of the following outcome indicators).
Our exclusion criteria include, first, no relevant outcome indicators; second, repeated publications, incomplete data, and second, repetitive, and incomplete data. It should be noted that incomplete data refer to per-protocol analysis instead of intention-to-treat analysis; and third, the intervention measures are Cinobufacin vs chemotherapy, or the control group is not chemotherapy alone.

The outcome indicators included in our study mainly involve clinical efficacy, KPS score, pain relief rate, tumor markers (CA125, CA153, and CEA), and adverse reactions (gastrointestinal reaction, myelosuppression, alopecia, liver and kidney damage, and peripheral neurotoxicity). The clinical efficacy includes overall response rate (ORR) and clinical benefit rate $(\mathrm{CBR})-\mathrm{ORR}=\mathrm{CR}+\mathrm{PR} /$ total cases $\times 100 \%$ and $\mathrm{CBR}=\mathrm{CR}+\mathrm{PR}+\mathrm{SD} /$ total cases $\times 100 \%[11]$.

2.4. Data Extraction. We first use EndNote to search and remove duplicate documents and read the remaining documents in depth. Two researchers (JX and KXD) independently screened the literature, extracted data, and crosschecked according to the inclusion and exclusion criteria. For those in doubt, they will be discussed or decided by a third researcher (DYL). The data extracted by this research include first author, year of publication, age, number of cases in each group, intervention measures, course of treatment, outcome indicators, etc.

2.5. Quality Evaluation. Two researchers (KXD and JW) used the risk bias assessment tool in the Cochrane evaluation manual [12] to evaluate the quality of the included literature and then cross-checked it. The evaluation criteria include random sequence generation, allocation concealment, blinding participants and personnel, blinding of outcome assessment, incomplete outcome data, selective reporting, and other bias. Disagreements are resolved through discussion or consultation with a third evaluator (DYL).

2.6. Statistical Analysis. We used RevMan5.3 software to conduct meta-analysis, heterogeneity test, sensitivity analysis and publication bias test on the included studies. The significance level is set to $\alpha=0.05$, and the heterogeneity is quantitatively analyzed by $I^{2}$. If $P<0.05, I^{2} \geq 50 \%$, there is obvious heterogeneity between the results of each study, and the random effect model is used for analysis. Mean difference (MD) is used for those with the same measurement unit, and relative risk (RR) and 95\% confidence interval (95\% CI) are used for binary classification variables. If the clinical heterogeneity is obvious, then the subgroup analysis or sensitivity analysis should be used for treatment.

\section{Results}

3.1. Search Results. We initially retrieved 163 related literature studies, and through reading the title, abstract, and full text of the literature studies, excluding animal experiments, repeated studies, and reviews, we finally met the inclusion criteria of 16 RCTs (Figure 1). 


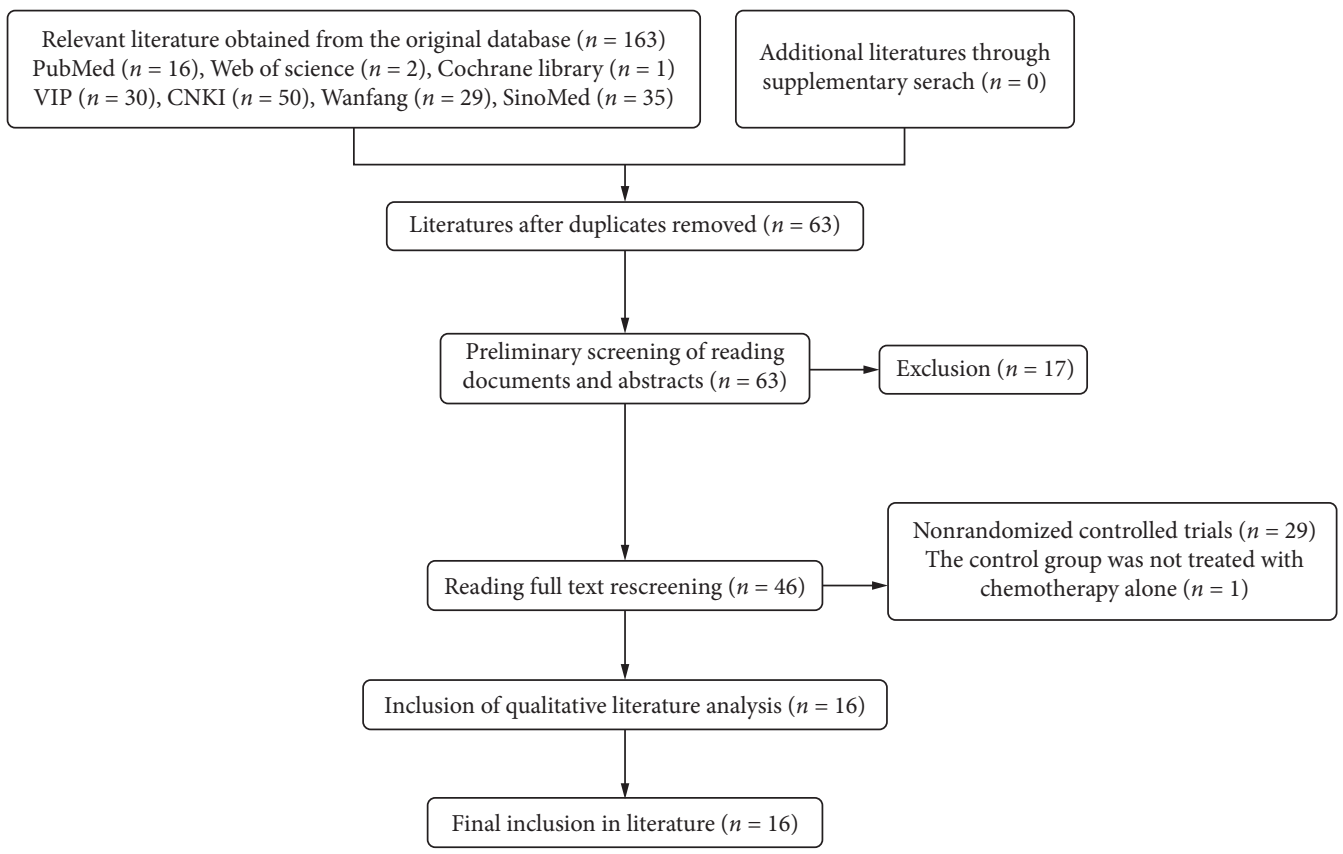

Figure 1: Flowchart of literature screening.

3.2. Basic Characteristics of Included Studies. The 16 literature studies [13-28] included in our study included 1331 patients, of which 666 patients in the Cinobufacin combined ith chemotherapy group and 665 patients in the chemotherapy-alone group. All the subjects were adult women, and the baseline of each study was comparable. The time span included in the study was 18 years. It should be pointed out that there are no randomized controlled trials of Cinobufacin in breast cancer treatment in foreign literature. The related studies Cinobufacin and breast cancer are reviews or related mechanism studies (Table 1).

3.3. Quality Evaluation of Included Studies. Nine of the 16 studies in our study reported specific random sequence generation methods, among which three studies [17, 18, 21] were grouped according to the order of admission or time and were rated as high-risk bias, while six studies $[13,16,20,26-28]$ were rated as low-risk bias, and the remaining seven studies only mentioned random and did not report the implementation of a specific random scheme. One study [20] was randomly grouped using the sealed envelope method, and no studies reported the implementation of the blind method. None of the studies reported the concealment of random allocation. Sixteen studies did not fully report the predetermined indicators, and there were cases where the results were selectively reported (Figure 2).

\subsection{Meta-Analysis Results}

3.4.1. Clinical Efficacy. The clinical efficacy of our research includes ORR (overall response rate) and CBR (clinical benefit rate). The 16 studies [13-28] (1331 cases) we included all reported ORR. The meta-analysis results found that the ORR of Cinobufacin combined with chemotherapy was superior to simple chemotherapy $(\mathrm{RR}=1.35,95 \% \mathrm{CI}$ : [1.23, 1.49], $P<0.00001)$. We included 15 studies [14-28] (1239 cases) reported CBR. The results of meta-analysis showed that the CBR of Cinobufacin combined with chemotherapy was better than that of chemotherapy alone $(\mathrm{RR}=1.14,95 \%$ CI: $[1.08,1.21], P<0.00001$ ) (Figure 3 ).

3.4.2. KPS Score and Pain Relief Rate. Five studies $[13,19,21,22,25]$ (368 cases) reported KPS scores, and three studies $[17,19,22]$ (153 cases) reported pain relief rate. The results of meta-analysis showed that the KPS score and pain relief rate of the Cinobufacin combined with chemotherapy group were better than those of chemotherapy-alone group, the RR of KPS scores was $1.98,95 \%$ CI was 1.45 to 2.68 , $P$-value was less than 0.0001 , and the RR of pain relief rate was $1.34,95 \% \mathrm{CI}$ was 1.01 to $1.78, P=0.04$ (Figures 4 and 5).

3.4.3. Tumor Markers. The six studies [13, 14, 18, 20, 26, 28] were reported the changes of CA153 and CEA, of which 4 studies $[14,18,20,26]$ reported changes in CA125. Through a comparative analysis of tumor markers before and after treatment in 1372 patients, we found that the heterogeneity between CA125, CA153, and CEA groups before treatment was relatively small and the fixed effects model was selected for meta-analysis. The results showed that there was no statistical difference in tumor markers before treatment $(P>0.05)$ (Figure 6). After treatment, the heterogeneity among CA125, CA153, and CEA groups was large and the random effects model was selected for meta-analysis. The results showed that the tumor markers of Cinobufacin combined with chemotherapy after treatment were lower than those of chemotherapy alone, and the difference was statistically significant; the MD of CA125 was $-7.36,95 \% \mathrm{CI}$ was -10.92 to $-3.80, P$-value was less than 0.0001 , the MD of 
TABLE 1: Basic characteristics of 16 included studies.

\begin{tabular}{|c|c|c|c|c|c|}
\hline $\begin{array}{l}\text { Author and } \\
\text { Year }\end{array}$ & $\begin{array}{c}\text { Treatment } \\
\text { Course (weeks) }\end{array}$ & Age $(\mathrm{T} / \mathrm{C})$ & $\begin{array}{r}\text { Number } \\
(\mathrm{T} / \mathrm{C})\end{array}$ & Interventions $(\mathrm{T} / \mathrm{C})$ & Outcome Indicators \\
\hline $\begin{array}{l}\text { Chen } 2019 \\
{[13]}\end{array}$ & $6 w$ & $\begin{array}{c}46.34 \pm 7.88 / \\
45.67 \pm 7.91\end{array}$ & $46 / 46$ & Cinobufacin $+\mathrm{NX} / \mathrm{NX}$ & $\mathrm{A} 1 \mathrm{C} 2 \mathrm{C} 3$ \\
\hline $\begin{array}{l}\text { Guo } 2019 \\
{[14]}\end{array}$ & $8 w$ & $\begin{array}{l}45.37 \pm 7.68 / \\
45.54 \pm 7.82\end{array}$ & $60 / 60$ & Cinobufacin + pirarubicin/Pirarubicin & $\mathrm{A} 1 \mathrm{~A} 2 \mathrm{C} 1 \mathrm{C} 2 \mathrm{C} 3 \mathrm{D} 1 \mathrm{D} 4$ \\
\hline $\begin{array}{l}\text { Lei } 2019 \\
{[15]}\end{array}$ & $6 w$ & $\begin{array}{l}50.42 \pm 3.62 / \\
50.46 \pm 3.68\end{array}$ & $29 / 29$ & Cinobufacin + DC/DC & A1A2D1D2D 5 \\
\hline $\begin{array}{l}\text { He } 2018 \\
{[16]}\end{array}$ & $8 w$ & $\begin{array}{l}43.7 \pm 1.9 / \\
44.33 \pm 2.1\end{array}$ & $68 / 68$ & Cinobufacin + TEC/TEC & $\mathrm{A} 1 \mathrm{~A} 2 \mathrm{D} 1$ \\
\hline $\begin{array}{l}\text { Wang } 2018 \\
{[17]}\end{array}$ & $6 w$ & Median age 54 & $35 / 35$ & Cinobufacin + DC/DC & A1A2B2D1D2D5 \\
\hline $\begin{array}{l}\text { Tian } 2017 \\
{[18]}\end{array}$ & $9-18 w$ & $\begin{array}{r}50.27 \pm 6.23 / \\
49.57 \pm 5.86 \\
\end{array}$ & $31 / 31$ & $\begin{array}{l}\text { Cinobufacin + basic scheme containing capecitabine/ } \\
\text { Basic scheme containing capecitabine }\end{array}$ & $\mathrm{A} 1 \mathrm{~A} 2 \mathrm{C} 1 \mathrm{C} 2 \mathrm{C} 3$ \\
\hline $\begin{array}{l}\text { Ke } 2017 \\
{[19]}\end{array}$ & $12 \mathrm{w}$ & $\begin{array}{l}\text { 38. } 3 \pm 8.9 / 39 \\
5 \pm 8.8\end{array}$ & $20 / 21$ & Cinobufacin $+\mathrm{CAF} / \mathrm{CAF}$ & $\mathrm{A} 1 \mathrm{~A} 2 \mathrm{~B} 1 \mathrm{~B} 2 \mathrm{D} 1 \mathrm{D} 3 \mathrm{D} 4$ \\
\hline $\begin{array}{l}\text { Deng } 2017 \\
{[20]}\end{array}$ & $6 w$ & $\begin{array}{c}50.27 \pm 6.23 / \\
49.57 \pm 5.86 \\
\end{array}$ & $31 / 31$ & $\begin{array}{l}\text { Cinobufacin + basic scheme containing capecitabine/ } \\
\text { Basic scheme containing capecitabine }\end{array}$ & $\mathrm{A} 1 \mathrm{~A} 2 \mathrm{C} 1 \mathrm{C} 2 \mathrm{C} 3$ \\
\hline $\begin{array}{l}\text { Yang } 2016 \\
{[21]}\end{array}$ & $8 w$ & $34.58 \pm 14.44$ & $40 / 41$ & Cinobufacin + TAC/TAC & A1A2B1D1D2D3 \\
\hline $\begin{array}{l}\text { Dong } 2011 \\
{[22]}\end{array}$ & $6 w$ & Average age 53 & $20 / 22$ & Cinobufacin + DC/DC & $\mathrm{A} 1 \mathrm{~A} 2 \mathrm{~B} 1 \mathrm{~B} 2 \mathrm{D} 1 \mathrm{D} 2 \mathrm{D} 5$ \\
\hline $\begin{array}{l}\text { Pan } 2011 \\
{[23]}\end{array}$ & $6 w$ & Average age 56 & $80 / 80$ & Cinobufacin + CAF/CAF & $\mathrm{A} 1 \mathrm{~A} 2 \mathrm{D} 1 \mathrm{D} 2 \mathrm{D} 4$ \\
\hline $\begin{array}{l}\text { Song } 2002 \\
{[24]}\end{array}$ & $4 w$ & Median age 54 & $26 / 21$ & Cinobufacin + CAF/CAF & $\mathrm{A} 1 \mathrm{~A} 2 \mathrm{D} 1 \mathrm{D} 2 \mathrm{D} 3$ \\
\hline $\begin{array}{l}\text { Sun } 2019 \\
{[25]}\end{array}$ & $6 w$ & $\begin{array}{l}41.85 \pm 2.25 / \\
43.56 \pm 3.02\end{array}$ & $56 / 56$ & Cinobufacin + TEC/TEC & A1A2B1D1D2D3 \\
\hline Li 2019 [26] & $9 w$ & $\begin{array}{l}49.34 \pm 7.34 / \\
48.36 \pm 8.52\end{array}$ & $30 / 30$ & $\begin{array}{c}\text { Cinobufacin + Basic scheme containing capecitabine/ } \\
\text { Basic scheme containing capecitabine }\end{array}$ & $\mathrm{A} 1 \mathrm{~A} 2 \mathrm{C} 1 \mathrm{C} 2 \mathrm{C} 3 \mathrm{D} 1$ \\
\hline $\begin{array}{l}\text { Mai } 2019 \\
{[27]}\end{array}$ & $6 w$ & $\begin{array}{l}44.10 \pm 2.92 / \\
43.50 \pm 3.71\end{array}$ & $25 / 25$ & $\begin{array}{c}\text { Cinobufacin }+ \text { docetaxel sequential CEF/Docetaxel } \\
\text { sequential CEF }\end{array}$ & A1A2D1D3 \\
\hline Li 2018 [28] & - & $\begin{array}{c}47.5 \pm 11.2 / \\
48.5 \pm 10.8\end{array}$ & $69 / 69$ & $\begin{array}{l}\text { Cinobufacin + pemetrexed combined with DDP/ } \\
\text { Pemetrexed combined with DDP }\end{array}$ & $\mathrm{A} 1 \mathrm{~A} 2 \mathrm{C} 2 \mathrm{C} 3 \mathrm{D} 1 \mathrm{D} 3 \mathrm{D} 4$ \\
\hline
\end{tabular}

Notes: T/C: treatment group/control group. A1(ORR); A2(CBR); B1(KPS score); B2(pain relief rate); C1(CA125); C2(CA153); C3(CEA); D1(gastrointestinal reaction); D2(myelosuppression); D3(liver and kidney damage); D4(alopecia); D5(peripheral neurotoxicity). TAC: docetaxel + pirarubicin + cyclophosphamide; TEC: docetaxel + epirubicin + cyclophosphamide; NX: capecitabine + vinorelbine; CAF: cyclophosphamide + pirarubicin + 5-FU; DC: docetaxel + capecitabine; CEF: cyclophosphamide + epirubicin + 5-FU.

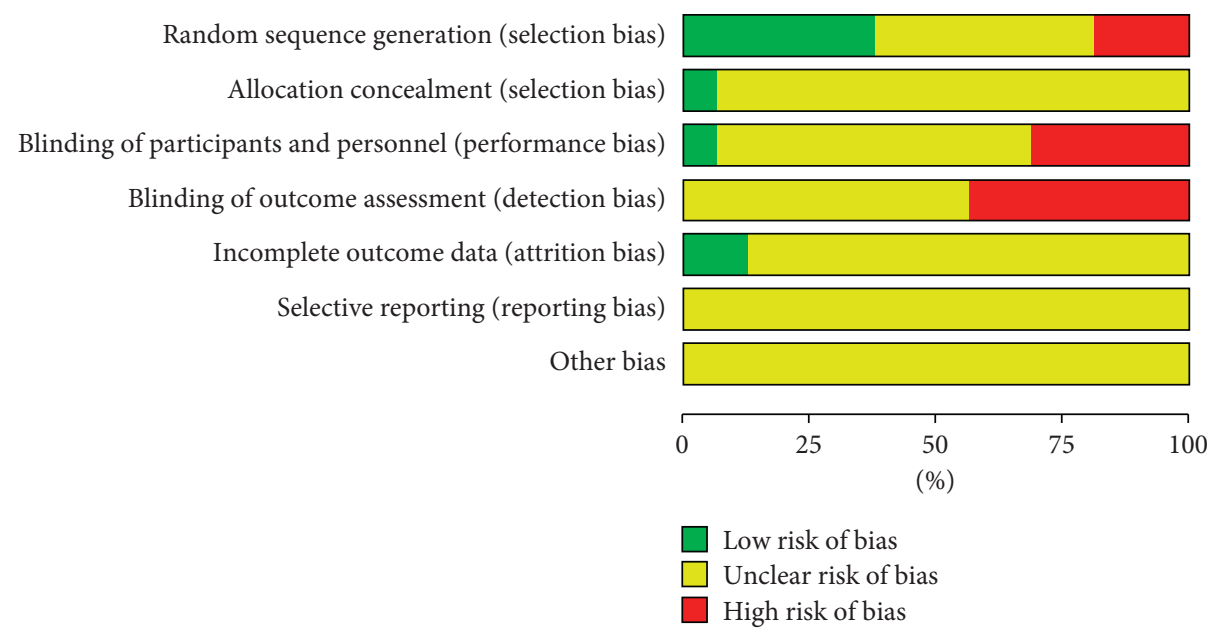

Figure 2: The diagram of risk of bias in included studies. 


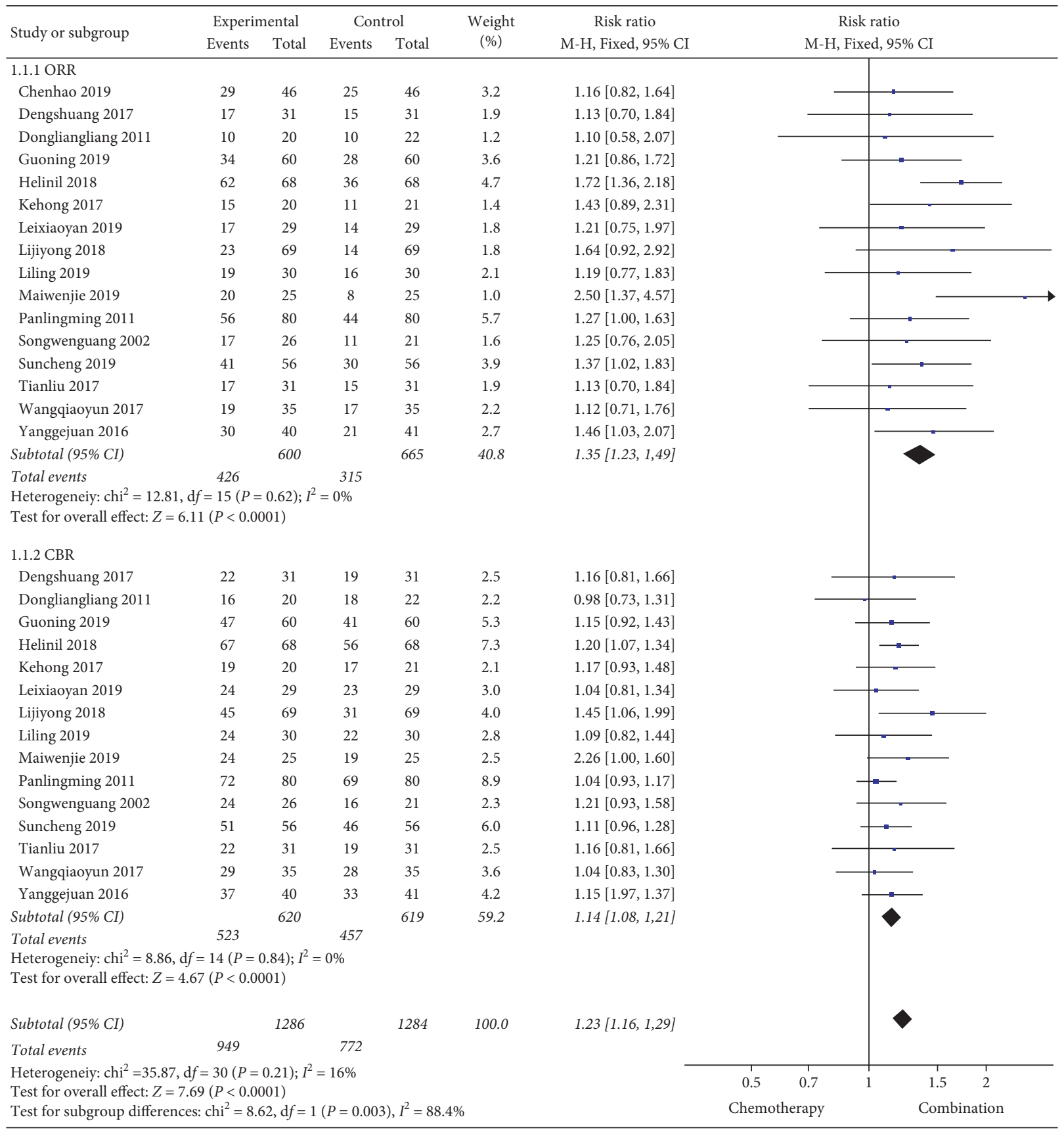

Figure 3: A meta-analysis of chemotherapy combined with Cinobufacin and chemotherapy alone for clinical efficacy.

CA153 was $-5.20,95 \%$ CI was -7.36 to $-3.03, P$-value was less than 0.00001 , and the MD of CEA was $-2.47,95 \%$ CI was -3.31 to $-1.62, P$-value was less than 0.00001 (Figure 7 ).

3.4.4. Adverse Reactions. 14 studies reported adverse reactions, including gastrointestinal reactions, liver and kidney damage, hair loss, peripheral neurotoxicity, bone marrow suppression, and so on. According to the heterogeneity test, the heterogeneity of gastrointestinal reaction, liver and kidney damage, hair loss, and peripheral neurotoxicity was small, and hence the fixed effects model was used for meta- analysis, while the heterogeneity of bone marrow suppression study was large, and hence the random effects model was used for meta-analysis. The results of meta-analysis showed that the incidences of gastrointestinal reaction, liver and kidney damage, and hair loss in the Cinobufacin combined with chemotherapy group were lower than those in the chemotherapy-alone group, and the RR of gastrointestinal reaction was $0.58,95 \% \mathrm{CI}$ was 0.48 to $0.70, P$-value was less than 0.00001 , the RR of liver and kidney damage was $0.57,95 \%$ CI was 0.38 to $0.84, P=0.004$, and the RR of hair loss was $0.61,95 \%$ CI was 0.40 to $0.92, P=0.02$. The incidences of peripheral neurotoxicity and myelosuppression 


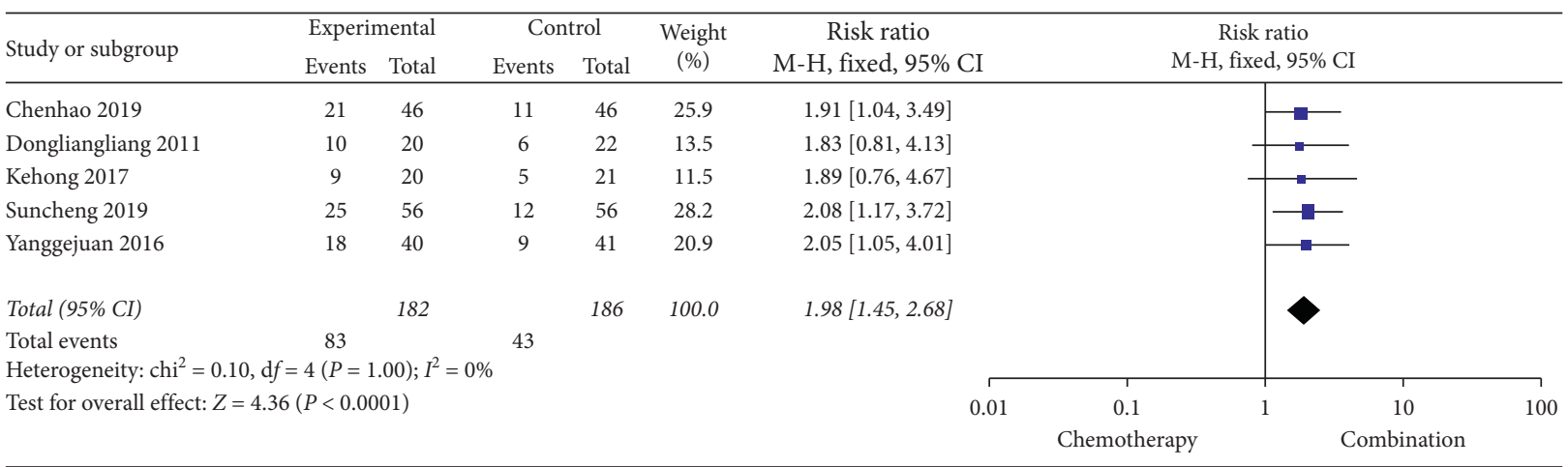

FIgURE 4: A meta-analysis of chemotherapy combined with Cinobufacin and chemotherapy alone for KPS scores after treatment.

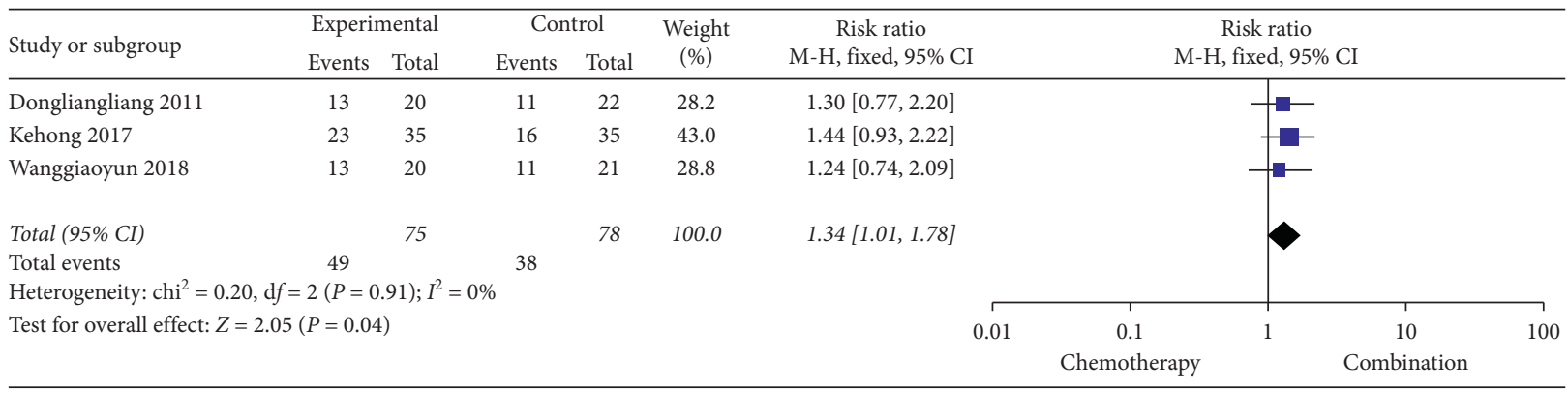

FIgURE 5: A meta-analysis of chemotherapy combined with Cinobufacin and chemotherapy alone for pain relief rate.

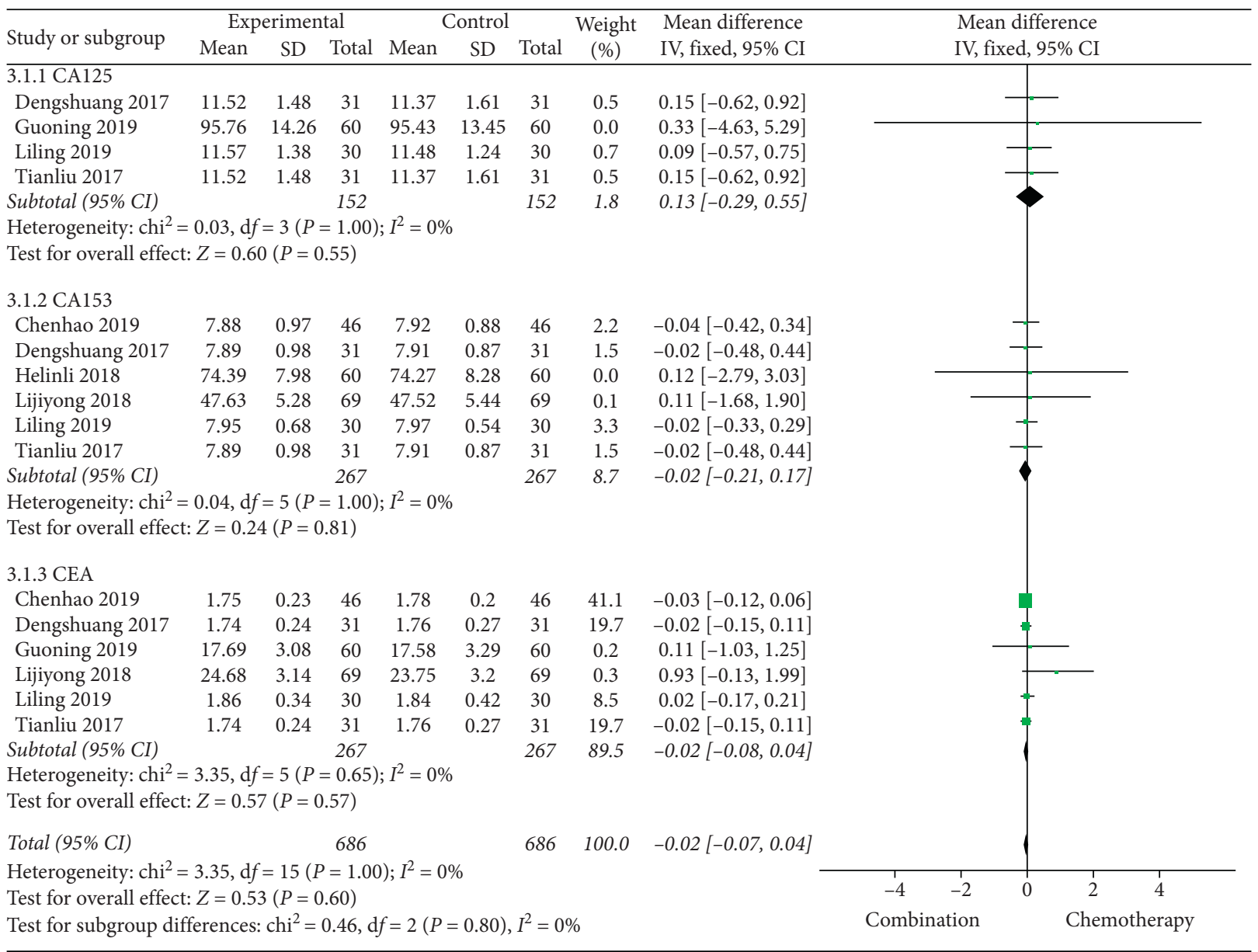

Figure 6: A meta-analysis of chemotherapy combined with Cinobufacin capsules and chemotherapy alone in tumor markers (CA125, CA153, and CEA) before treatment. 


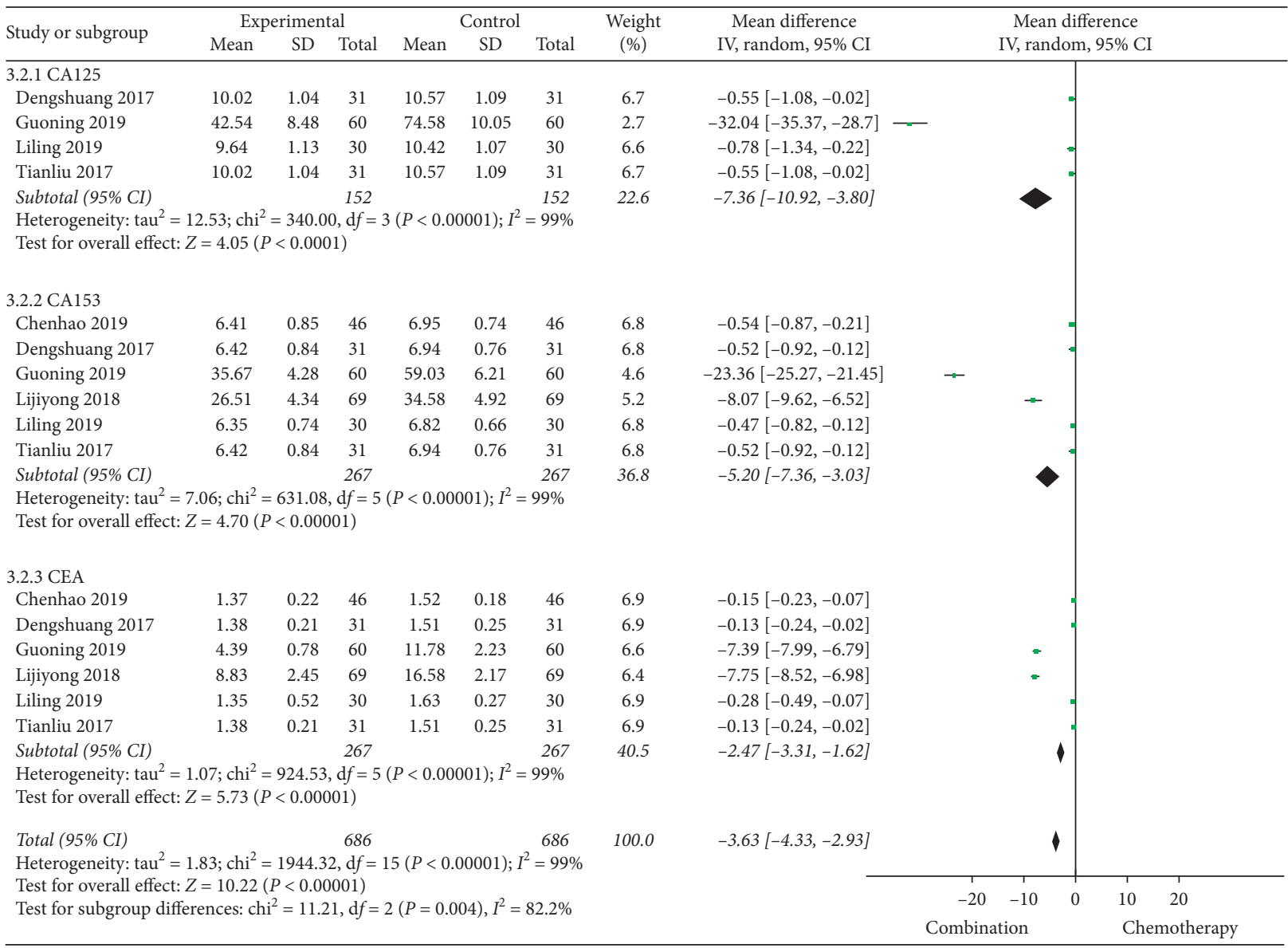

FIgURe 7: A meta-analysis of chemotherapy combined with Cinobufacin capsules and chemotherapy alone in tumor markers (CA125, CA153, and CEA) after treatment.

in the combination group were similar to those in the chemotherapy group, and the difference was not statistically significant, the RR of peripheral neurotoxicity was $0.69,95 \%$ CI was 0.26 to $1.85, P$-value was 0.46 , and the RR of myelosuppression was $0.78,95 \% \mathrm{CI}$ was 0.46 to $1.34, P=$ 0.37 (Figures 8 and 9).

3.5. Sensitivity Analysis. Our results showed that the heterogeneity of tumor markers (CA125, CA153, and CEA) after treatment was relatively large $\left(I^{2}=99 \%\right)$. After the comparative analysis was included in the literature, the heterogeneity of tumor markers after treatment was significantly reduced $\left(I^{2}=0\right)$ after the removal of the studies by Guo et al. [14] and Li [28], so we considered that the heterogeneity of tumor markers after treatment was mainly related to the quality of the included study (Figure 10).

3.6. Publication Bias Analysis. The funnel chart analysis of clinical efficacy showed that the results were not completely symmetrical, which was related to the low quality of the study and the small sample size (Figure 11).

\section{Discussion}

In this study, the meta-analysis method was used to merge and analyze 16 randomized controlled literature studies on the efficacy and safety of Cinobufacin combined with chemotherapy for breast cancer. The results of meta-analysis showed that the ORR, CBR, KPS scores, and pain relief rate of the Cinobufacin combined with chemotherapy group were better than those of the chemotherapy-alone group, which suggested that Cinobufacin combined with chemotherapy could improve the clinical efficacy and quality of life of the patients with breast cancer. Our study also found that the tumor markers (CA125, CA153, and CEA) of the Cinobufacin combined with chemotherapy group were lower than those of the chemotherapy-alone group. The heterogeneity was related to the low-quality literature of the included studies, but the results were stable. In terms of safety, the incidences of gastrointestinal reactions, liver and kidney damage, and hair loss in the Cinobufacin combined chemotherapy group were lower than those in the simple chemotherapy group, and the difference was statistically significant, but the incidences of peripheral neurotoxicity and myelosuppression in the combined group were similar 


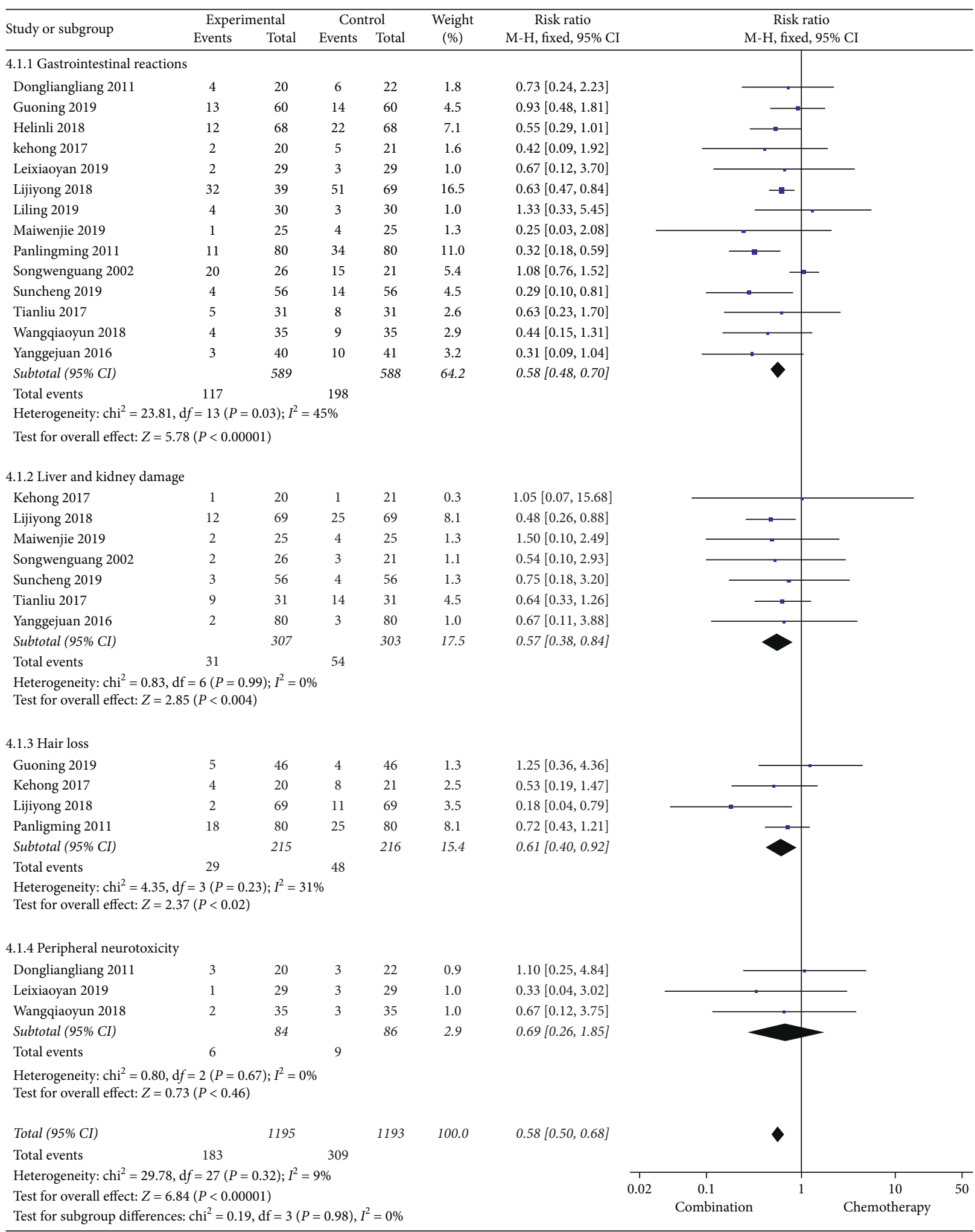

FIgURE 8: A meta-analysis of chemotherapy combined with Cinobufacin and chemotherapy alone for adverse reactions.

to those in the chemotherapy group, and the difference was not statistically significant.

Chemotherapy is the most common and direct clinical treatment for advanced breast cancer, but it usually requires large doses of two or more chemotherapeutic agents, which will bring corresponding side effects when reaching the treatment dose. The minor side effects will have a certain impact on the daily life of the patients, and the severe toxicities will threaten the physical and mental health of the patients, which will lead to the failure of chemotherapy and 


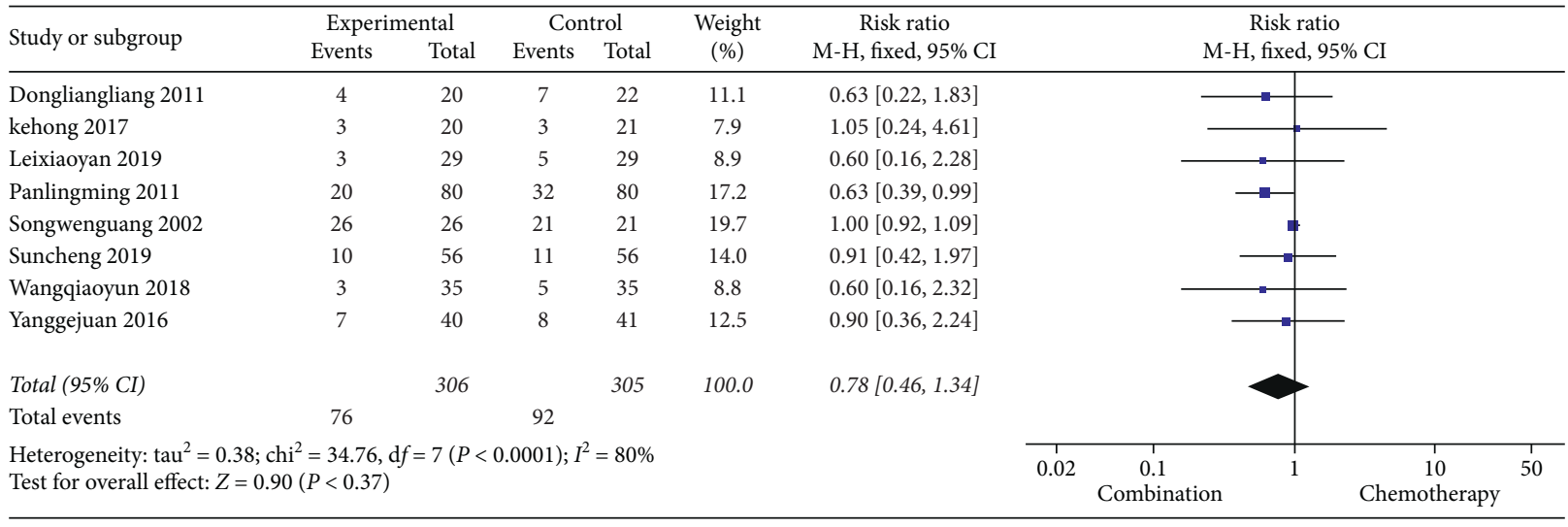

FIgURE 9: A meta-analysis of chemotherapy combined with Cinobufacin and chemotherapy alone in myelosuppression.

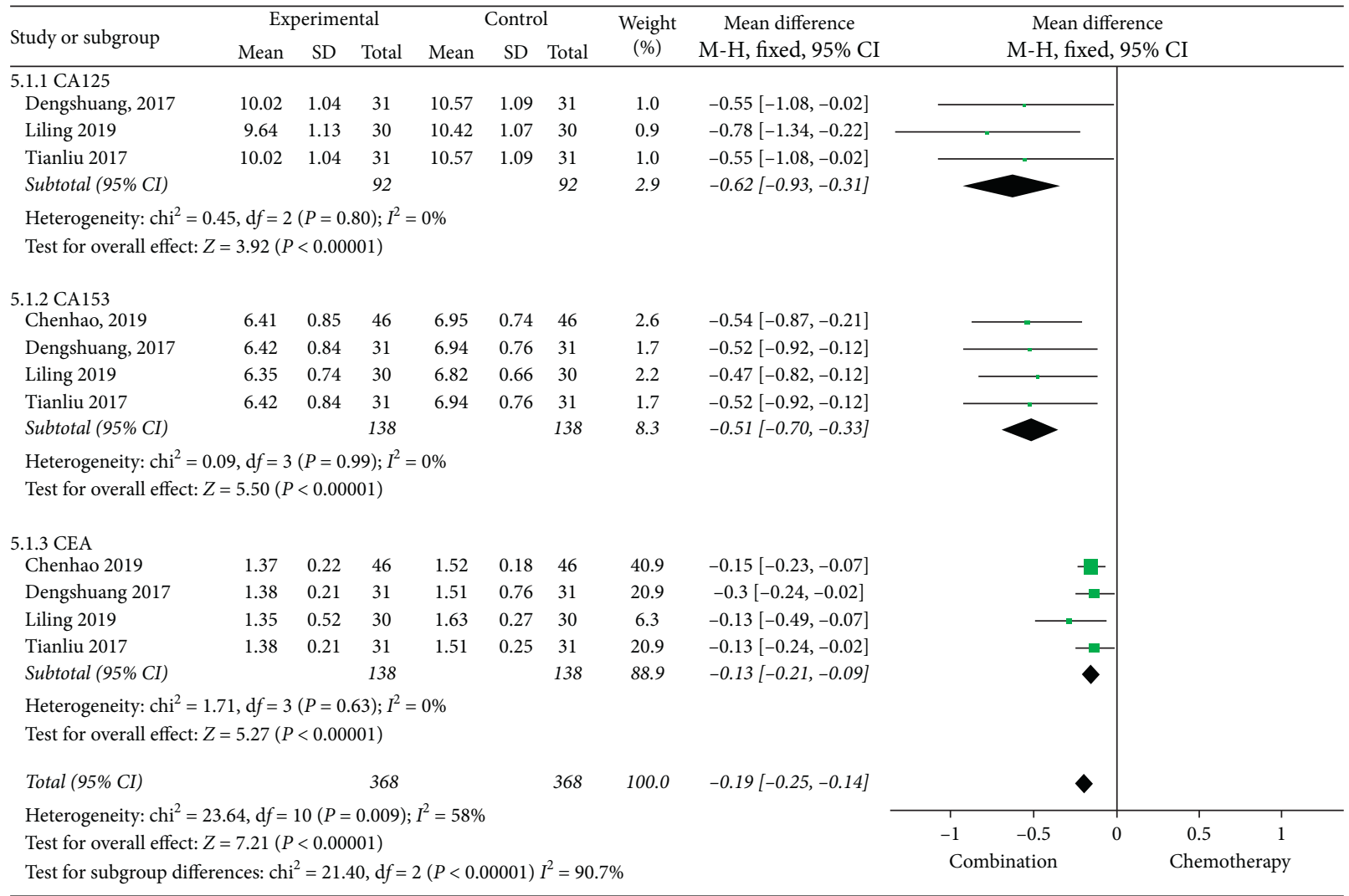

FIGURE 10: A meta-analysis of sensitivity analysis of chemotherapy combined with Cinobufacin and chemotherapy alone in tumor markers (CA125, CA153, and CEA) after treatment.

affect the prognosis [29-31]. Therefore, the combination of traditional Chinese and Western medicine has become a common clinical treatment for malignant tumors [32]. As the incidence of breast cancer increases year by year, an antitumor traditional Chinese medicine preparation independently developed by China, Cinobufacin, can be used in the field of breast cancer in combination with the chemotherapeutics to reduce the adverse reactions of patients and improve the quality of life of patients [33]. The mechanism may be as follows: First, apoptosis of human breast cancer cell line T-47D can be induced by increasing the expression level of caspase-3 [34]; second, apoptosis of MDA-MB-231 can be induced by destroying the cytoskeleton, resulting in abnormal changes of the cell surface ultrastructure and morphology [35]; Third, it can inhibit the growth and proliferation of human breast cancer cell lines MDA-MB468 and BT549 by inhibiting their proliferation and migration and the activity of PI3K/Akt signaling pathway $[36,37]$; Fourth, many studies have shown that due to a large number of fibrin accumulation and platelet aggregation 


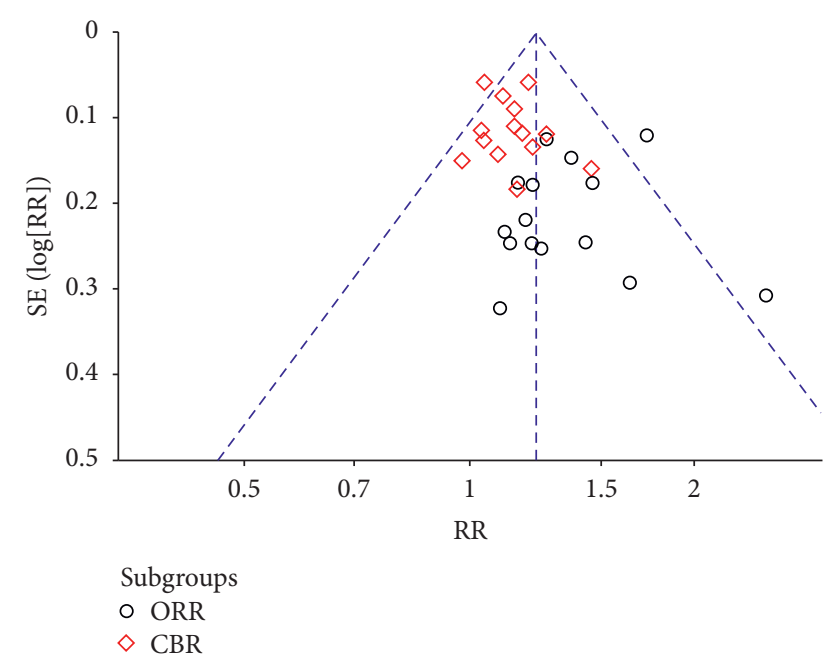

FIgURE 11: Funnel chart of clinical efficacy.

around cancer cells, patients with malignant tumors are prone to coagulation dysfunction, which makes the blood present a hypercoagulable state, while traditional Chinese medicine for promoting blood circulation and removing blood stasis can expose cancer cells, so they are more vulnerable to the attack of chemotherapy drugs [38, 39]. Therefore, the efficacy of Cinobufacin in clearing away heat and detoxification, promoting blood circulation, and removing stasis can play a role of "increasing efficiency and reducing toxicity" when combined with chemotherapy.

At present, a large number of experimental studies have found that Cinobufacin has antitumor effects on breast cancer, lung cancer, esophageal cancer, gastric cancer, liver cancer, bladder cancer, etc. [40]. Ni et al. [41] treated MGC-803 and BGC-823 GC cells with different concentrations of Cinobufacin and found that Cinobufacin has significant antitumor cell proliferation and apoptosis effects both in vivo and in vitro and can inhibit the growth of gastric cancer cells by inhibiting the Akt/ mTOR pathway and induce cell apoptosis through the internal pathway. Yin et al. [42] established a nude mouse xenograft model and found that Cinobufacin can inhibit the growth of liver metastases by reducing the expression of MMP-2, MMP-9 and VEGF. Yang et al. [43] also found that Cinobufacin can inhibit the growth of human bladder cancer cells in vivo and in vitro through Fas/Fasl and TNF- $\alpha$ /TNFR1 pathways. Not only experimental studies have confirmed the scientific antitumor effect of Cinobufacin but also a large number of clinical studies have confirmed the rationality of its clinical application. Sha et al. [44] through the RCT test on the effect of Cinobufacin capsule combined with raltitrexed and oxaliplatin on advanced colorectal cancer found that Cinobufacin capsule combined with raltitrexed and oxaliplatin can enhance the immune function of patients with advanced colorectal cancer, reduce tumor markers, and inhibit the growth and metastasis of tumor cells and neovascularization. Wang et al. [45] found that patients with advanced liver cancer who were treated with Cinobufacin as a single agent had a lower rate of disease deterioration $(11.4 \%)$ and a higher total effective rate after treatment $(82.86 \%)$. Serum total bilirubin and alanine aminotransferase decreased significantly.

Our study also found that Cinobufacin combined with chemotherapy can reduce the incidences of gastrointestinal reactions, liver and kidney damage, and alopecia in breast cancer patients, and there are also relevant clinical reports. Dong [46] found that the incidences of nausea, vomiting, and leukopenia were lower in the combined group than in the control group after randomly dividing 68 patients with advanced colon cancer into the chemotherapy group and chemotherapy combined with Cinobufacin group. Cao, et al. [47] found that the combined group could reduce the incidence of myelosuppression and improve the disease control rate through the RCT trial of Cinobufacin injection combined with first-line chemotherapy in the treatment of advanced non-small-cell lung cancer. In addition, studies on adverse reactions related to Cinobufacin itself have mainly focused on reports of Cinobufacin injection. Cheng [48] retrospectively analyzed 272 cases of adverse reactions/ events of Cinobufacin injection and found that the adverse reactions produced by Cinobufacin injection were mainly rapid-onset type, which mainly manifested as rapid-onset skin reactions in addition to venous injury and other adverse reactions. Zhang [49] analyzed 60 cases of adverse reactions caused by Cinobufacin injection and found that the main reactions were allergic and febrile reactions, and no deaths were observed.

Our study is a comprehensive analysis of the RCT literature of Cinobufacin for breast cancer, but there are still some deficiencies. First, the chemotherapy methods used in the studies included in this study are not the same. Although they are all patients with advanced breast cancer, the pathological stage is not exactly the same, which increases the clinical heterogeneity of the study.

Second, blind methods and random allocation concealment were not implemented in some of the included studies, which made the quality of the included studies low. Third, although the Chinese and English databases were searched extensively, the included cases were still from China. Finally, this article has not yet carried out stratified analysis of different dosage forms of Cinobufacin, which may increase the heterogeneity of the study. By consulting the relevant literature, we found that the main components of the three formulations were dried toad skin extract, which could play an antitumor role by inhibiting the growth and reproduction of tumor cells, inducing cell apoptosis and antimetastasis, targeting $\mathrm{Na}^{+} / \mathrm{K}^{+}$-ATPase activity, inhibiting tumor angiogenesis, and inhibiting the steroid receptor coactivator family [50].

\section{Conclusion}

In summary, the results of this study indicate that Cinobufacin combined with chemotherapy can improve the clinical efficacy of breast cancer patients, enhance the quality of life of the patients, reduce the value of tumor markers such as CA125, CA153, and CEA, and lower the occurrence 
of adverse reactions such as gastrointestinal reactions, liver and kidney damage, and hair loss. However, the conclusion of safety should be used carefully especially, the corresponding adverse reactions caused by different dosage forms of Cinobufacini itself should be considered. In future, we will carry out more clinical studies on different dosage forms of Cinobufacin and further compare the efficacy of different dosage forms and the same chemotherapy regimen in the treatment of breast cancer.

\section{Abbreviations}

CNKI: Chinese National Knowledge Infrastructure

RCT: randomized controlled trials

ORR: overall response rate

CBR: clinical benefit rate

KPS: Karnofsky Performance Status

CR: complete response

PR: partial response

SD: $\quad$ stable disease

RR: relative risk

CI: confidence interval

MD: mean difference.

\section{Data Availability}

All the data were taken from the published studies.

\section{Conflicts of Interest}

The authors declare that there are no conflicts of interest between them.

\section{Authors' Contributions}

JX was mainly responsible for research design, literature retrieval, data extraction, data analysis, and writing of papers; DYL was involved in research design, research design, data verification, and quality evaluation; KXD was involved in data extraction and quality evaluation; and JW was involved in research design, quality evaluation, and thesis implication and modification. The author read and approved the final manuscript.

\section{Acknowledgments}

The authors wish to thank Ms. Elizabeth Gullen from Yale University for the critical reading of the manuscript. This work was supported by the Key Program (81630080) and Young Scientist Fund (81503575) of National Natural Science Foundation of China, and Young Scientist Development Program 2016 of Dongzhimen Hospital Affiliated to Beijing University of Chinese Medicine (DZMYS-201610).

\section{References}

[1] M. Ghoncheh, Z. Pournamdar, and H. Salehiniya, "Incidence and mortality and epidemiology of breast cancer in the world," Asian Pacific Journal of Cancer Prevention, vol. 17, no. sup3, pp. 43-46, 2016.
[2] F. Bray, J. Ferlay, I. Soerjomataram, R. L. Siegel, L. A. Torre, and A. Jemal, "Global cancer statistics 2018: GLOBOCAN estimates of incidence and mortality worldwide for 36 cancers in 185 countries," CA: A Cancer Journal for Clinicians, vol. 68, no. 6, pp. 394-424, 2018.

[3] G. Miolo, E. Muraro, D. Martorelli et al., "Anthracycline-free neoadjuvant therapy induces pathological complete responses by exploiting immune proficiency in HER2+ breast cancer patients," BMC Cancer, vol. 15, no. 14, p. 954, 2014.

[4] Z. P. Gong, T. Chen, L. R. Deng et al., "Clinical application progress of cinobufagin to cancer pain relief," Drugs \& Clinical, vol. 25, no. 4, pp. 268-271, 2010.

[5] S. Kai, J.-H. Lu, P.-P. Hui, and H. Zhao, "Pre-clinical evaluation of cinobufotalin as a potential anti-lung cancer agent," Biochemical and Biophysical Research Communications, vol. 452, no. 3, pp. 768-774, 2014.

[6] Y. Xu, D. Han, F. C. Feng et al., "Meta-analysis of cinobufacini injection combined with platinum-contained first-line chemotherapy in meta-analysis of cinobufacini Injection combined with platinum-contained first-line chemotherapy in treatment of non-small cell lung cancer," China Journal of Chinese Materia Medica, vol. 44, no. 21, pp. 4728-4737, 2019.

[7] Z. Y. Dong, X. T. Qiu, S. A. Kujawa et al., "Cinobufacini injection for moderate and advanced primary liver cancer: a systematic review and meta- analysis," Journal of Chinese Pharmaceutical Sciences, vol. 28, no. 4, pp. 264-275, 2019.

[8] H. Sun, W. Wang, M. Bai, and D. Liu, "Cinobufotalin as an effective adjuvant therapy for advanced gastric cancer: a metaanalysis of randomized controlled trials," OncoTargets and Therapy, vol. 12, no. 26, pp. 3139-3160, 2019.

[9] Y. Y. Peng, Z. Chen, F. Y. Wang et al., "Meta analysis of cinobufacin capsule combined with oxaliplatin chemotherapy in the treatment of colorectal cancer," Acta Chinese Medicine, vol. 35, no. 3, pp. 673-678, 2020.

[10] D. Moher, A. Liberati, J. Tetzlaff, D. G. Altman, and PRISMA Group, "Preferred reporting items for systematic reviews and meta-analyses: the PRISMA statement," International Journal of Surgery, vol. 8, no. 5, pp. 336-341, 2010.

[11] E. A. Eisenhauer, P. Therasse, J. Bogaerts et al., "New response evaluation criteria in solid tumours: revised RECIST guideline (version 1.1)," European Journal of Cancer, vol. 45, no. 2, pp. 228-247, 2009.

[12] H. Steglich-Arnholm, M. Holtmannspötter, C. Gluud et al., "Carotid artery stenting versus no stenting assisting thrombectomy for acute ischaemic stroke: protocol for a systematic review of randomised clinical trials with meta-analyses and trial sequential analyses," Systematic Reviews, vol. 5, no. 1, p. 208, 2016.

[13] H. Chen, "Effect of huachansu capsule combined with NX chemotherapy on serum related tumor markers and median survival in patients with advanced breast cancer," Hebei Medicine, vol. 25, no. 3, pp. 533-537, 2019.

[14] N. Guo, C. X. Li, D. M. Zhu et al., "Clinical study of Huachansu Capsules combined with pirarubicin in treatment of advanced breast cancer," Drugs \& Clinical, vol. 34, no. 1, pp. 200-204, 2019.

[15] X. Y. Lei, "To explore the clinical effect of Cinobufacin injection combined with chemotherapy in the treatment of advanced breast cancer," World Latest Medicine Information, vol. 19, no. 5, p. 132, 2019.

[16] L. L. He and B. Zhou, "Efficacy and safety of cinobufacini capsules combined with systemic chemotherapy in treatment of patients with middle-advanced breast cancer," Evaluation 
and Analysis of Drug-Use in Hospitals of China, vol. 18, no. 3, pp. 334-336, 2018.

[17] Q. Y. Wang and Z. Lu, "The clinical efficacy of cinobufacini injection combined chemotherapy in the treatment of advanced breast cancer," China \& Foreign Medical Treatment, vol. 37, no. 6, pp. 125-127, 2018.

[18] L. Tian, "Effects and tolerance of Huachansu Capsule in aided treatment of advanced breast cancer," Medical Journal of National Defending Forces in Southwest China, vol. 27, no. 11, pp. 1173-1175, 2017.

[19] H. Ke, J. Cui, J. L. Jin et al., "Clinical analysis of cinobufotalin capsule combined CAF scheme in treating moderate and advanced breast cancer," World Chinese Medicine, vol. 12, no. 10, pp. 2358-2361, 2017.

[20] S. Deng, G. B. Feng, and J. D. Xu, "Curative effects and patients' tolerance of cinobufotalin capsules combined with capecitabine based regimens in the treatment of advanced breast cancer," Pharmaceutical and Clinical Research, vol. 25, no. 5, pp. 439-443, 2017.

[21] G. J. Yang, X. H. Wang, R. Pang et al., "Analysis of the efficacy of cinobufotalin injection combined with systemic chemotherapy in the treatment of middle-advanced breast cancer," Progress in Modern Biomedicine, vol. 16, no. 23, pp. 44784480, 2016.

[22] L. L. Dong and L. M. Zhang, "Clinical observation of cinobutacini injection combined with chemotherapy used to treat advanced breast cancer," China Medicine and Pharmacy, vol. 1, no. 14, pp. 93-94, 2011.

[23] L. M. Pan, "Analysis of 160 cases of advanced breast cancer combining cinobufacin and chemotherapy," China \& Foreign Medical Treatment, vol. 30, no. 1, pp. 59-61, 2011.

[24] W. G. Song, R. L. Wang, and Z. Z. Fu, "Clinical observation of Cinobufacin combined with chemotherapy in the treatment of advanced breast cancer," Chinese Journal of Integrative Medicine, vol. 13, pp. 158-160, 2002.

[25] C. Sun, Y. C. Liu, and W. Zhao, "Clinical effect of cinobufotalin Injection combined with systemic chemotherapy in the treatment of advanced breast cancer," China Modern Medicine, vol. 26, no. 20, pp. 43-45, 2019.

[26] L. Li, "Therapeutic effect of cinobufotalin capsule combined with capecitabine-containing basic regimen in the treatment of advanced breast cancer and its influence on related serum indicators," Contemporary Medicine, vol. 26, no. 1, pp. 91-93, 2020.

[27] W. J. Mai, Y. X. Fu, K. Ji et al., "Clinical observation on cinobufotalin capsule combined with Docetaxel in treatment of breast cancer," Chinese Archives of Traditional Chinese Medicine, vol. 37, no. 8, pp. 1959-1962, 2019.

[28] J. Y. Li, "Efficacy and safety of pemetrexed combined with cinobufacini in the first-line treatment of advanced or metastatic breast cancer," Chinese Archives of General Surger$y$ (Electronic Edition), vol. 12, no. 1, pp. 32-35, 2018.

[29] R. Kaaks, T. Johnson, K. Tikk et al., "Insulin-like growth factor $\mathrm{I}$ and risk of breast cancer by age and hormone receptor status-A prospective study within the EPIC cohort," International Journal of Cancer, vol. 134, no. 11, pp. 2683-2690, 2014.

[30] K. Tikk, D. Sookthai, T. Johnson et al., "Circulating prolactin and breast cancer risk among pre- and postmenopausal women in the EPIC cohort," Annals of Oncology, vol. 25, no. 7, pp. 1422-1428, 2014.

[31] M. Brackstone, D. Palma, A. B. Tuck et al., "Concurrent neoadjuvant chemotherapy and radiation therapy in locally advanced breast cancer," International Journal of Radiation Oncology*Biology*Physics, vol. 99, no. 4, pp. 769-776, 2017.

[32] L. K. Xing, J. Wang, Y. Zhang et al., "Cinobufotalin combined with chemotherapy for the advanced gastrointestinal cancer: a Meta-analysis," Chinese Journal of Gastroenterology and Hepatology, vol. 25, no. 7, pp. 779-786, 2016.

[33] M. McCulloch, C. See, X.-J. Shu et al., "Astragalus-based Chinese herbs and platinum-based chemotherapy for advanced non-small-cell lung cancer: meta-analysis of randomized trials," Journal of Clinical Oncology, vol. 24, no. 3, pp. 419-430, 2006.

[34] W. Wang, A. Shi, and Z. Fan, "Apoptosis of T-47D cells induced by cinobufacini via a caspase-3-dependent manner," Chemical Research in Chinese Universities, vol. 30, no. 1, pp. 108-113, 2014.

[35] L. Ma, B. Song, H. Jin et al., "Cinobufacini induced MDA-MB231 cell apoptosis-associated cell cycle arrest and cytoskeleton function," Bioorganic \& Medicinal Chemistry Letters, vol. 22, no. 3, pp. 1459-1463, 2012.

[36] X. A. He, J. J. Lv, and M. L. Zou, "Clinical research of neoadjuvant chemothempy with Docetaxel plus epirubicin in patients with advanced breast cancer," The Journal of Medical Theory and Practice, vol. 27, no. 15, pp. 1973-1977, 2014.

[37] J. J. Mou, Study on the Effect and Mechanism of Cinobufaine on Breast Cancer, Dalian Medical University, Dalian, China, 2015.

[38] J. J. Lv, "To analyze the effect of huachansu tablets on coagulation status, treatment effect and quality of life of patients with advanced lung cancer," Guide of China Medicine, vol. 17, no. 12, p. 194, 2019.

[39] X. S. Xiao, "Clinical observation of 50 cases of intermediate and advanced liver cancer treated with huachansu intervention chemotherapy," China \& Foreign Medical Treatment, vol. 30, no. 25, p. 80, 2011.

[40] X. Y. Wu, F. Tian, X. J. Zhu et al., "Progress of Cinobufacini Antitumor Research," Research and Practice on Chinese Medicines, vol. 32, no. 5, pp. 82-86, 2018.

[41] T. Ni, H. Wang, D. Li et al., "Huachansu Capsule inhibits the proliferation of human gastric cancer cells via Akt/mTOR pathway," Biomedicine \& Pharmacotherapy, vol. 118, Article ID 109241, 2019.

[42] J. H. Yin, X. Y. Zhu, W. D. Shi et al., "Huachansu injection inhibits metastasis of pancreatic cancer in mice model of human tumor xenograft," BMC Complementary and Alternative Medicine, vol. 14, p. 483, 2014.

[43] T. Yang, R. Shi, L. Chang et al., "Huachansu suppresses human bladder cancer cell growth through the fas/fasl and TNF- alpha/TNFR1 pathway in vitro and in vivo," Journal of Experimental \& Clinical Cancer Research, vol. 34, no. 1, p. 21, 2015.

[44] X. F. Sha, Z. F. Song, J. Ding et al., "Effects of Huachansu capsules combined with raltitrexed and olisaplatin on advanced colorectal cancer immunity, tumor markers, matrix metalloproteinases and angiogenesis," Journal of Hainan Medical University, vol. 24, no. 23, pp. 2066-2069, 2018.

[45] Z. C. Wang, Z. P. Feng, and H. Wang, "Short-term efficacy oF cinobufacini in the treatment oF advanced liver cancer," Modern Hospitals, vol. 8, no. 6, pp. 56-57, 2008.

[46] M. Dong, "Observation on the clinical efficacy of cinobufotalin combined with chemotherapy in the treatment of advanced colon cancer," Chinese Community Doctors, vol. 34, no. 13, pp. 27-28, 2018.

[47] J. Cao, J. Zhou, D. Yang et al., "Clinical curative effect on nonsmall cell lung cancer patients by cinobufacini injection 
combined first-line chemotherapy," Journal of International Oncology, vol. 43, no. 10, pp. 741-743, 2016.

[48] M. Chen, "Analysis on 272 cases of adverse reactions/incidents induced by cinobufacini injection," China Pharmaceuticals, vol. 22, no. 16, pp. 71-72, 2013.

[49] D. Q. Zhang, "Causes of adverse reactions caused by cinobufacin injection and analysis of rational drug use," AntiInfection Pharmacy, vol. 14, no. 4, pp. 807-809, 2017.

[50] C. S. Cheng, J. Wang, J. Chen et al., "New therapeutic aspects of steroidal cardiac glycosides: the anticancer properties of HuaChanSu and its main active constituent bufalin," Cancer Cell International, vol. 19, p. 92, 2019. 